\title{
Two-Weight Norm Inequality for the One-Sided Hardy-Littlewood Maximal Operators in Variable Lebesgue Spaces
}

\author{
Caiyin Niu, ${ }^{1,2}$ Zongguang Liu, ${ }^{1}$ and Panwang Wang' \\ ${ }^{1}$ School of Science, China University of Mining \& Technology, Beijing 100083, China \\ ${ }^{2}$ Faculty of Mathematics and Physics, Huaiyin Institute of Technology, Huaian, Jiangsu 223003, China \\ Correspondence should be addressed to Caiyin Niu; niucaiyin@yahoo.com
}

Received 24 June 2016; Revised 16 October 2016; Accepted 27 October 2016

Academic Editor: Stanislav Hencl

Copyright (c) 2016 Caiyin Niu et al. This is an open access article distributed under the Creative Commons Attribution License, which permits unrestricted use, distribution, and reproduction in any medium, provided the original work is properly cited.

The authors establish the two-weight norm inequalities for the one-sided Hardy-Littlewood maximal operators in variable Lebesgue spaces. As application, they obtain the two-weight norm inequalities of variable Riemann-Liouville operator and variable Weyl operator in variable Lebesgue spaces on bounded intervals.

\section{Introduction and Main Results}

The one-sided Hardy-Littlewood maximal operators $M^{+}$and $M^{-}$are defined by

$$
\begin{aligned}
& M^{+} f(x)=\sup _{h>0} \frac{1}{h} \int_{x}^{x+h}|f(t)| \mathrm{d} t, \\
& M^{-} f(x)=\sup _{h>0} \frac{1}{h} \int_{x-h}^{x}|f(t)| \mathrm{d} t .
\end{aligned}
$$

Sawyer [1] showed that $M^{+}$is bounded from $L^{p}(v)$ to $L^{p}(u)$ if the pairs of nonnegative functions $(u, v)$ satisfy Sawyer-type two-weight condition for the one-sided maximal operator. In [2] Martín-Reyes et al. generalized this result to $M_{g}^{+}$, where

$$
M_{g}^{+} f(x)=\sup _{h>0} \frac{1}{\int_{x}^{x+h} g(t) \mathrm{d} t} \int_{x}^{x+h}|f(t)| g(t) \mathrm{d} t
$$

with $g$ is positive locally integrable function. The similar results are also true for $M^{-}$and $M_{g}^{-}$(see [1-3]).
Let $E$ be a measurable set in $\mathbb{R}$. Given a measurable function $p(\cdot): E \rightarrow(0,+\infty)$, we denote

$$
\begin{aligned}
& p_{E}^{-}=\underset{x \in E}{\operatorname{ess} \inf _{x}} p(x), \\
& p_{E}^{+}=\underset{x \in E}{\operatorname{ess} \sup _{x \in}} p(x) .
\end{aligned}
$$

If $E=\mathbb{R}$, we will simply note that $p^{-}=p_{\mathbb{R}}^{-}$and $p^{+}=p_{\mathbb{R}}^{+}$.

Definition 1. Given $p(\cdot): E \rightarrow[1,+\infty), \sigma$ is a locally integrable function such that $0<\sigma(x)<\infty$ a.e. $x \in E$. We say that $p(\cdot) \in \mathscr{P}_{\sigma}^{+}(E)$ if there exists a constant $C>0$ such that for every $(x, x+h) \subset E$, with $\int_{x}^{x+h} \sigma(t) \mathrm{d} t \leqslant 1 / 2$,

$$
p(x)-p_{(x, x+h)}^{-} \leqslant \frac{C}{-\ln \left(\int_{x}^{x+h} \sigma(t) \mathrm{d} t\right)} .
$$

Definition 2 (see [4]). It is given that $p(\cdot): E \rightarrow[1,+\infty)$. We say that $p(\cdot) \in L H_{\infty}(E)$, if there exist constants $p_{\infty} \geqslant 1$ and $C_{\infty}>0$ such that for all $x \in E$

$$
\left|p(x)-p_{\infty}\right| \leqslant \frac{C_{\infty}}{\ln (e+|x|)} .
$$


Let $1 \leqslant p_{E}^{-} \leqslant p_{E}^{+}<\infty$. The variable Lebesgue space $L^{p(\cdot)}(E)$ is the set of measurable functions $f$ on $E$ such that

$$
\rho_{p(\cdot), E}(f):=\int_{E}|f(x)|^{p(x)} \mathrm{d} x<\infty
$$

This is a Banach space (see [4-7]) with the norm

$$
\|f\|_{L^{p(\cdot)}(E)}=\inf \left\{\lambda>0: \rho_{p(\cdot), E}\left(\frac{f}{\lambda}\right) \leqslant 1\right\} .
$$

If $E=\mathbb{R}$, we will write $\|f\|_{L^{p(\cdot)}(E)}$ as $\|f\|_{p(\cdot)}$. The variable Lebesgue space is the special case of the Musielak-Orlicz space (see $[7,8])$. For the detail of $L^{p(\cdot)}$ we refer to [4-7] and so on.

Let $w$ be a nonnegative locally integrable function on $E$. The weighted variable Lebesgue space $L_{w}^{p(\cdot)}(E)$ is the set of measurable functions $f$ on $E$ such that $f w \in L^{p(\cdot)}(E)$ and $\|f\|_{L_{w}^{p^{(\cdot)}(E)}}=\|f w\|_{L^{p(\cdot)}(E)}$. When $p(\cdot)=p$ is a constant, $L_{w}^{p(\cdot)}(E)$ coincides with the classical weighted Lebesgue space $L^{P}\left(w^{p}\right)(E)$.

Edmunds et al. [9] investigated the boundedness of $M^{+}$ and $M^{-}$in the variable Lebesgue spaces $L^{p(\cdot)}$. The two-weight weak type modular inequalities of $M^{+}$and $M^{-}$in $L^{p(\cdot)}$ were discussed in [10]. In [11], Kokilashvili et al. acquired the sufficient condition such that $M^{+}$and $M^{-}$are bounded from $L_{w}^{p(\cdot)}\left(\mathbb{R}_{+}\right)$to $L_{v}^{p(\cdot)}\left(\mathbb{R}_{+}\right)$, where $p(\cdot)$ is constant on some interval $(a,+\infty)(a>0)$ and $T_{v, w}$ is bounded in $L^{p(\cdot)}\left(\mathbb{R}_{+}\right)$with

$$
T_{v, w} f(x)=v(x) \int_{x}^{+\infty} \frac{f(y)}{y w(y)} \mathrm{d} y, \quad x \in \mathbb{R}_{+} .
$$

Throughout this paper, $u$ and $v$ are nonnegative locally integrable functions and $C$ is a positive constant whose value may change from one occurrence to the next. For exponent function $p(\cdot)$ with $p(x) \geqslant 1$, its conjugate exponent will be denoted by $p^{\prime}(\cdot)$ with $1 / p(x)+1 / p^{\prime}(x)=1$. For a Lebesgue measurable set $E \subset \mathbb{R}, \chi_{E}$ will be its characteristic function.

Definition 3. It is given that $p(\cdot): E \rightarrow[1,+\infty)$ such that $1 \leqslant p_{E}^{-} \leqslant p_{E}^{+}<\infty$. We say that $(u, v) \in S_{p(\cdot)}^{+}(E)$ if there exists a constant $C>0$ such that for every interval $I=(a, b) \subset E$, with $\int_{-\infty}^{a} u(x)^{p(x)} \mathrm{d} x>0$,

$$
\begin{aligned}
& \int_{I} M^{+}\left(\chi_{I} \sigma\right)(x)^{p(x)} u(x)^{p(x)} \mathrm{d} x \leqslant C \int_{I} \sigma(x) \mathrm{d} x \\
& \quad<+\infty
\end{aligned}
$$

where $v(x)>0$ a.e. $x \in E$ and $\sigma(x)=v(x)^{-p^{\prime}(x)}$.

Definition 4. It is given that $p(\cdot): E \rightarrow[1,+\infty)$ such that $1 \leqslant p_{E}^{-} \leqslant p_{E}^{+}<\infty$. We say that $(u, v) \in S_{p(\cdot)}^{++}(E)$ if there exist constants $m \geqslant 1 / p_{E}^{-}$and $C_{1}, C_{2}>0$ such that for every interval $I=(a, b) \subset E$, with $\int_{-\infty}^{a} u(x)^{p(x)} \mathrm{d} x>0$,

$$
\begin{gathered}
\int_{I} M^{+}\left(\chi_{I} \sigma\right)(x)^{p(x)} \frac{u(x)^{p(x)}}{(e+|x|)^{m p_{E}^{-}}} \mathrm{d} x \\
\leqslant C_{1} \int_{I} \frac{\sigma(x)}{(e+|x|)^{m p_{E}^{-}}} \mathrm{d} x \leqslant C_{2},
\end{gathered}
$$

where $v(x)>0$ a.e. $x \in E$ and $\sigma(x)=v(x)^{-p^{\prime}(x)}$.

$S_{p(\cdot)}^{+}(E)$ and $S_{p(\cdot)}^{+}(E)$ can be considered to be the generalization of Sawyer-type two-weight condition (see [1]) for the one-sided maximal operator in variable exponents case. If $E=\mathbb{R}$ we will simply write $S_{p(\cdot)}^{+}(\mathbb{R})$ as $S_{p(\cdot)}^{+}$and $S_{p(\cdot)}^{++}(\mathbb{R})$ as $S_{p(\cdot)}^{\prime+}$. We can define $p(\cdot) \in \mathscr{P}_{\sigma}^{-}(E),(u, v) \in S_{p(\cdot)}^{-}(E)$, and $(u, v) \in S_{p(\cdot)}^{-}(E)$ in similar ways.

Our main results are the following theorems.

Theorem 5. Let $D=(-\infty, d), d<+\infty$, and $p(\cdot): D \rightarrow$ $[1,+\infty)$ such that $1<p_{D}^{-} \leqslant p_{D}^{+}<+\infty$. If $(u, v) \epsilon$ $\left(S_{p(\cdot)}^{+}(D) \cap S_{p(\cdot)}^{++}(D)\right)$ and $p(\cdot) \in\left(\mathscr{P}_{\sigma}^{+}(D) \cap L H_{\infty}(D)\right)$ with $\sigma(x)=v(x)^{-p^{\prime}(x)}$, then

$$
\left\|\left(M^{+} f\right) u\right\|_{L^{p(\cdot)}(D)} \leqslant C\|f v\|_{L^{p(\cdot)}(D)} .
$$

Theorem 6. Let $D=(d,+\infty), d>-\infty$, and $p(\cdot): D \rightarrow$ $[1,+\infty)$ such that $1<p_{D}^{-} \leqslant p_{D}^{+}<+\infty$. If $(u, v) \in$ $\left(S_{p(\cdot)}^{+}(D) \cap S_{p(\cdot)}^{++}(D)\right)$ and $p(\cdot) \in\left(\mathscr{P}_{\sigma}^{+}(D) \cap L H_{\infty}(D)\right)$ with $\sigma(x)=v(x)^{-p^{\prime}(x)}$, then

$$
\left\|\left(M^{+} f\right) u\right\|_{L^{p(\cdot)}(D)} \leqslant C\|f v\|_{L^{p(\cdot)}(D)} .
$$

Theorem 7. Let $D$ be a bounded interval and let $p(\cdot): D \rightarrow$ $[1,+\infty)$ such that $1<p_{D}^{-} \leqslant p_{D}^{+}<+\infty$. If $(u, v) \in S_{p(\cdot)}^{+}(D)$ and $p(\cdot) \in \mathscr{P}_{\sigma}^{+}(D)$ with $\sigma(x)=v(x)^{-p^{\prime}(x)}$, then

$$
\left\|\left(M^{+} f\right) u\right\|_{L^{p(\cdot)}(D)} \leqslant C\|f v\|_{L^{p(\cdot)}(D)} .
$$

Corollary 8. It is given that $p(\cdot): \mathbb{R} \rightarrow[1,+\infty)$ such that $1<p^{-} \leqslant p^{+}<+\infty$. If there exists a constant $c>0$ such that $(u, v) \in\left(S_{p(\cdot)}^{+} \cap S_{p(\cdot)}^{\prime+}(\mathbb{R} \backslash[-c, c])\right)$ and $p(\cdot) \in\left(\mathscr{P}_{\sigma}^{+}(\mathbb{R}) \cap L H_{\infty}(\mathbb{R} \backslash\right.$ $[-c, c]))$ with $\sigma(x)=v(x)^{-p^{\prime}(x)}$, then

$$
\left\|\left(M^{+} f\right) u\right\|_{p(\cdot)} \leqslant C\|f v\|_{p(\cdot)} .
$$

Corollary 9. It is given that $p(\cdot): \mathbb{R} \rightarrow[1,+\infty)$ such that $1<p^{-} \leqslant p^{+}<+\infty,(u, v) \in S_{p(\cdot)}^{+}$, and $p(\cdot) \in \mathscr{P}_{\sigma}^{+}(\mathbb{R})$ with $\sigma(x)=v(x)^{-p^{\prime}(x)}$. If

(a) there exists a constant $c>0$ such that $p(\cdot) \in L H_{\infty}(\mathbb{R} \backslash$ $[-c, c])$ and $p(x) \geqslant p_{\infty}$ for $x \in(\mathbb{R} \backslash[-c, c])$,

(b) there exist constants $m \geqslant 1 / p^{-}$and $C>0$ such that for every interval $I=(a, b) \subset(\mathbb{R} \backslash[-c, c])$, with $\int_{-\infty}^{a} u(x)^{p(x)} \mathrm{d} x>0$,

$$
\int \frac{\sigma(x)}{(e+|x|)^{m p^{-}}} \mathrm{d} x \leqslant C .
$$


Then

$$
\left\|\left(M^{+} f\right) u\right\|_{p(\cdot)} \leqslant C\|f v\|_{p(\cdot)} .
$$

Remark 10. In Theorems 5 and 6, the set $S_{p(\cdot)}^{+}(D) \cap S_{p(\cdot)}^{++}(D)$ is not empty. In fact, if $v(x) \geqslant 1$ and $0 \leqslant u(x) \leqslant v(x)^{1 /(1-p(x))}$, when $x \in D$, then $(u, v) \in\left(S_{p(\cdot)}^{+}(D) \cap S_{p(\cdot)}^{++}(D)\right)$.

Remark 11. If we change the conditions $S_{p(\cdot)}^{+}, S_{p(\cdot)}^{++}, \mathscr{P}_{\sigma}^{+}$to $S_{p(\cdot)}^{-}$, $S_{p(\cdot)}^{\prime-}$, and $\mathscr{P}_{\sigma}^{-}$, respectively, in above theorems, we will obtain similar results of $M^{-}$.

Remark 12. If we take $u(x)=1$ and $v(x)=1$ for a.e. $x \in D$ whenever $D$ is an open interval, then $\mathscr{P}_{\sigma}^{+}(D)=\mathscr{P}_{1}^{+}(D)=$ $\mathscr{P}^{+}(D)$ (see Definition 18 below) and the results of this paper coincide with those of [9].

Remark 13. The Sawyer-type condition was used earlier in [12] to characterize the two-weight boundedness of the classical Hardy-Littlewood maximal operator $M$. Corresponding results for variable Lebesgue spaces can be found in [13-15].

\section{Proof of the Main Results}

In order to establish our main results, we will need following lemmas.

Lemma 14 (see $[4])$. It is given that $E$ and $p(\cdot): E \rightarrow[1,+\infty)$ such that $p_{E}^{+}<\infty$.

(a) If $\|f\|_{L^{p(\cdot)}(E)} \leqslant 1$, then $\rho_{p(\cdot), E}(f)^{1 / p_{E}^{-}} \leqslant\|f\|_{L^{p(\cdot)}(E)} \leqslant$ $\rho_{p(\cdot), E}(f)^{1 / p_{E}^{+}}$.

(b) If $\|f\|_{L^{p(\cdot)}(E)}>1$, then $\rho_{p(\cdot), E}(f)^{1 / p_{E}^{+}} \leqslant\|f\|_{L^{p(\cdot)}(E)} \leqslant$ $\rho_{p(\cdot), E}(f)^{1 / p_{E}^{-}}$

In particular, if $\|f\|_{L^{p(\cdot)}(E)} \leqslant 1$, then $\rho_{p(\cdot), E}(f) \leqslant 1$.

Lemma 15 (see [4]). If $p_{E}^{+}<\infty$, the set of bounded functions with compact support is dense in $L^{p(\cdot)}(E)$.

Lemma 16 (see [1]). Suppose $g \geqslant 0$ is integrable function with compact support on $\mathbb{R}$. If $I=(a, b)$ is a component interval of the open set $\left\{x \in \mathbb{R}: M^{+} g(x)>\lambda\right\}$ with $\lambda>0$, then

$$
\frac{1}{b-x} \int_{x}^{b} g(t) \mathrm{d} t \geqslant \lambda \quad \text { for } a \leqslant x<b .
$$

Lemma 17 (see [16]). It is given that a set $G \subseteq \mathbb{R}$ and two exponents $s(\cdot)$ and $r(\cdot)$ such that

$$
|s(y)-r(y)| \leqslant \frac{C_{0}}{\ln (e+|y|)} .
$$

Then for every $t \geqslant 1$ there exists a constant $C=C\left(t, C_{0}\right)$ such that for all functions $f$ with $|f(y)| \leqslant 1$,

$$
\begin{aligned}
\int_{G}|f(y)|^{s(y)} \mathrm{d} \mu(y) \leqslant & C \int_{G}|f(y)|^{r(y)} \mathrm{d} \mu(y) \\
& +\int_{G} \frac{1}{(e+|y|)^{t r_{G}^{-}}} \mathrm{d} \mu(y),
\end{aligned}
$$

where $\mu$ is a given nonnegative measure.

Proof of Theorem 5. By the homogeneity, Lemma 15, the Fatou lemma for variable Lebesgue spaces [4] and Lemma 14, we only need to prove

$$
\int_{-\infty}^{d} M^{+} f(x)^{p(x)} u(x)^{p(x)} \mathrm{d} x \leqslant C
$$

for the nonnegative bounded function $f$ with compact support and $\|f v\|_{L^{p(\cdot)}(D)}=1$. Let $N>|d|$ be a positive integer and for $k \in \mathbb{Z}$, define

$$
\Omega_{k, N}=\left\{x \in \mathbb{R}: M^{+} f(x)>2^{k}\right\} \cap(-N, d) .
$$

Obviously each $\Omega_{k, N}$ is a bounded open set. Let $I_{j k}=\left(a_{j k}, b_{j k}\right)$ be the component intervals of $\Omega_{k, N}$, where $j$ is an integer. Applying Lemma 16 to a fixed $I_{j k}$, we have

$$
\frac{1}{b_{j k}-x} \int_{x}^{b_{j k}} f(t) \mathrm{d} t \geqslant 2^{k} \quad \text { for } x \in\left[a_{j k}, b_{j k}\right) .
$$

Let $E_{j k}=I_{j k} \backslash \Omega_{k+1, N}$, then the sets $\left\{E_{j k}\right\}$ are pairwise disjoint and for every $k$

$$
\bigcup_{j} E_{j k}=\left\{x \in \mathbb{R}: 2^{k}<M^{+} f(x) \leqslant 2^{k+1}\right\} \cap(-N, d) .
$$

Therefore

$$
\begin{aligned}
& \int_{-N}^{d} M^{+} f(x)^{p(x)} u(x)^{p(x)} \mathrm{d} x \\
& =\sum_{k, j} \int_{E_{j k}} M^{+} f(x)^{p(x)} u(x)^{p(x)} \mathrm{d} x \\
& \leqslant \sum_{k, j} \int_{E_{j k}}\left(2^{k+1}\right)^{p(x)} u(x)^{p(x)} \mathrm{d} x \\
& \leqslant 2^{p_{D}^{+}} \sum_{k, j} \int_{E_{j k}}\left(\frac{1}{b_{j k}-x} \int_{x}^{b_{j k}} f(t) \mathrm{d} t\right)^{p(x)} u(x)^{p(x)} \mathrm{d} x \\
& =2^{p_{D}^{+}} \sum_{k, j} \int_{-N}^{d}\left(A_{j k}\left(f \sigma^{-1}\right)(x)\right)^{p(x)} \varphi_{j k}(x) \mathrm{d} x,
\end{aligned}
$$


where

$$
\begin{gathered}
A_{j k} g(x) \\
=\left(\frac{1}{\int_{x}^{b_{j k}} \sigma(t) \mathrm{d} t} \int_{x}^{b_{j k}} g(t) \sigma(t) \mathrm{d} t\right) \chi_{E_{j k}}(x), \\
\varphi_{j k}(x) \\
=\left(\frac{1}{b_{j k}-x} \int_{x}^{b_{j k}} \sigma(t) \mathrm{d} t\right)^{p(x)} u(x)^{p(x)} \chi_{E_{j k}}(x) . \\
\text { We show that for every } j \text { and } k \text { the inequality } \\
\quad A_{j k} g(x)^{p(x)} \leqslant C\left(\left(A_{j k}\left(g^{p(\cdot) / p_{D}^{-}}\right)(x)\right)^{p_{D}^{-}}\right. \\
\left.+A_{j k} R(x)^{p_{D}^{-}}+R(x)^{p_{D}^{-}}\right)
\end{gathered}
$$

is valid, where $g=f \sigma^{-1}, R(x)=C /(e+|x|)^{m}\left(m \geqslant 1 / p_{D}^{-}\right.$is the same as that in (10)), and $C$ is independent of $j$ and $k$.

Let $q(x)=p(x) / p_{D}^{-}$, then $q(\cdot) \in\left(\mathscr{P}_{\sigma}^{+}(D) \cap L H_{\infty}(D)\right)$. By $\|f v\|_{L^{p(\cdot)}(D)}=1$ and Lemma 14 we get

$$
\int_{D} g(x)^{p(x)} \sigma(x) \mathrm{d} x=\int_{D} f(x)^{p(x)} v(x)^{p(x)} \mathrm{d} x \leqslant 1 .
$$

Let $g(x)=g_{1}(x)+g_{2}(x)$, where

$$
\begin{aligned}
& g_{1}(x)=g(x) \chi_{\{x \in D: g(x)>1\}}(x), \\
& g_{2}(x)=g(x) \chi_{\{x \in D: g(x) \leqslant 1\}}(x),
\end{aligned}
$$

then

$$
\begin{aligned}
& A_{j k} g(x)^{p(x)} \\
& \quad \leqslant 2^{p_{D}^{+}-1}\left(A_{j k} g_{1}(x)^{p(x)}+A_{j k} g_{2}(x)^{p(x)}\right) .
\end{aligned}
$$

Since $g_{1}(t) \geqslant 1$, by $(27)$ we can get

$$
\int_{x}^{b_{j k}} g_{1}(t) \sigma(t) \mathrm{d} t \leqslant \int_{D} g_{1}(t)^{p(t)} \sigma(t) \mathrm{d} t \leqslant 1
$$

for $x \in E_{j k}$. Then

$$
\begin{gathered}
A_{j k} g_{1}(x)^{q(x)} \leqslant\left(\int_{x}^{b_{j k}} \sigma(t) \mathrm{d} t\right)^{-q(x)} \\
\cdot\left(\int_{x}^{b_{j k}} g_{1}(t) \sigma(t) \mathrm{d} t\right)^{q_{\left(x, b_{j k}\right)}^{-}} \\
=\left(\int_{x}^{b_{j k}} \sigma(t) \mathrm{d} t\right)^{-\left(q(x)-q_{\left(x, b_{j k}\right)}^{-}\right)} \\
\cdot\left(\frac{1}{\int_{x}^{b_{j k}} \sigma(t) \mathrm{d} t} \int_{x}^{b_{j k}} g_{1}(t) \sigma(t) \mathrm{d} t\right)^{q_{\left(x, b_{j k}\right)}^{-}} .
\end{gathered}
$$

If $\int_{x}^{b_{j k}} \sigma(t) \mathrm{d} t \leqslant 1 / 2$, by (4), we have

$$
\begin{gathered}
\left(\int_{x}^{b_{j k}} \sigma(t) \mathrm{d} t\right)^{-\left(q(x)-q_{\left(x, b_{j k}\right)}^{-}\right)} \\
\leqslant\left(\int_{x}^{b_{j k}} \sigma(t) \mathrm{d} t\right)^{C / \ln \left(\int_{x}^{b_{j k}} \sigma(t) \mathrm{d} t\right)} \leqslant C . \\
\text { If } \int_{x}^{b_{j k}} \sigma(t) \mathrm{d} t>1 / 2 \text {, then }
\end{gathered}
$$

$$
\left(\int_{x}^{b_{j k}} \sigma(t) \mathrm{d} t\right)^{-\left(q(x)-q_{\left(x, b_{j k}\right)}^{-}\right)} \leqslant 2^{q(x)-q_{\left(x, b_{j k}\right)}^{-}} \leqslant 2^{q_{D}^{+}} \leqslant C .
$$

Therefore

$$
\begin{aligned}
& A_{j k} g_{1}(x)^{p(x)} \\
& \quad \leqslant C\left(\frac{1}{\int_{x}^{b_{j k}} \sigma(t) \mathrm{d} t} \int_{x}^{b_{j k}} g_{1}(t) \sigma(t) \mathrm{d} t\right)^{q_{\left(x, b_{j k}\right)}^{-} p_{D}^{-}} .
\end{aligned}
$$

Thus, by the Hölder inequality and $g_{1}(t) \geqslant 1$ we can get

$$
\begin{aligned}
& A_{j k} g_{1}(x)^{p(x)} \\
& \quad \leqslant C\left(\frac{1}{\int_{x}^{b_{j k}} \sigma(t) \mathrm{d} t} \int_{x}^{b_{j k}} g_{1}(t)^{q_{\left(x, b_{j k}\right)}^{-}} \sigma(t) \mathrm{d} t\right)^{p_{D}^{-}} \\
& \quad \leqslant C\left(\frac{1}{\int_{x}^{b_{j k}} \sigma(t) \mathrm{d} t} \int_{x}^{b_{j k}} g_{1}(t)^{q(t)} \sigma(t) \mathrm{d} t\right)^{p_{D}^{-}} \\
& \quad \leqslant C\left(A_{j k}\left(g^{p(\cdot) / p_{D}^{-}}\right)(x)\right)^{p_{D}^{-}} .
\end{aligned}
$$

Next, we will estimate $A_{j k} g_{2}(x)$. If $A_{j k} g_{2}(x) \leqslant R(x)$, by $R(x) \leqslant 1$, we have

$$
A_{j k} g_{2}(x)^{q(x)} \leqslant R(x)^{q(x)} \leqslant R(x) .
$$

On the other hand, if $A_{j k} g_{2}(x)>R(x)>0$, noticing that $A_{j k} g_{2}(x) \leqslant 1$ and

$$
q(x)-q_{\infty} \geqslant-\left|q(x)-q_{\infty}\right| \quad \text { for } x \in \mathbb{R}
$$

thus

$$
\begin{aligned}
0 & <A_{j k} g_{2}(x)^{q(x)-q_{\infty}} \leqslant A_{j k} g_{2}(x)^{-\left|q(x)-q_{\infty}\right|} \\
& \leqslant R(x)^{-\left|q(x)-q_{\infty}\right|} .
\end{aligned}
$$


Applying (38), $q(\cdot) \in L H_{\infty}(D)$ and the Hölder inequality,

$$
\begin{aligned}
& A_{j k} g_{2}(x)^{q(x)} \leqslant A_{j k} g_{2}(x)^{-\left|q(x)-q_{\infty}\right|} A_{j k} g_{2}(x)^{q_{\infty}} \\
& \leqslant R(x)^{-\left|q(x)-q_{\infty}\right|} \\
& \cdot\left(\frac{1}{\int_{x}^{b_{j k}} \sigma(t) \mathrm{d} t} \int_{x}^{b_{j k}} g_{2}(t) \sigma(t) \mathrm{d} t\right)^{q_{\infty}} \\
& \leqslant C\left(\int_{x}^{b_{j k}} \sigma(t) \mathrm{d} t\right)^{-1} \int_{x}^{b_{j k}} g_{2}(t)^{q_{\infty}} \sigma(t) \mathrm{d} t .
\end{aligned}
$$

By $0 \leqslant g_{2}(t) \leqslant 1$ and Lemma 17, we get

$$
\begin{aligned}
& A_{j k} g_{2}(x)^{q(x)} \leqslant C\left(\int_{x}^{b_{j k}} \sigma(t) \mathrm{d} t\right)^{-1} \\
& \quad\left(\int_{x}^{b_{j k}} g_{2}(y)^{q(y)} \sigma(y) \mathrm{d} y\right. \\
& \left.+\int_{x}^{b_{j k}} \frac{\sigma(y)}{(e+|y|)^{m q_{\left(x, b_{j k}\right)}^{-}}} \mathrm{d} y\right) \leqslant C\left(A_{j k}\left(g_{2}^{q(\cdot)}\right)(x)\right. \\
& \left.+A_{j k} R(x)\right) .
\end{aligned}
$$

Hence, combining (36) and (40)

$$
\begin{aligned}
A_{j k} g_{2}(x)^{p(x)} \leqslant C\left(A_{j k}\left(g_{2}^{q(\cdot)}\right)(x)+A_{j k} R(x)\right. \\
+R(x))^{p_{D}^{-}} \leqslant C\left(\left(A_{j k}\left(g^{p(\cdot) / p_{D}^{-}}\right)(x)\right)^{p_{D}^{-}}\right. \\
\left.\quad+A_{j k} R(x)^{p_{D}^{-}}+R(x)^{p_{D}^{-}}\right) .
\end{aligned}
$$

This completes the proof of (26) by (29), (35), and (41). Applying (26) to (24), we obtain

$$
\begin{aligned}
& \int_{-N}^{d} M^{+} f(x)^{p(x)} u(x)^{p(x)} \mathrm{d} x \\
& \leqslant C \sum_{k, j} \int_{-N}^{d}\left(A_{j k}\left(\left(f \sigma^{-1}\right)^{p(\cdot) / p_{D}^{-}}\right)(x)\right)^{p_{D}^{-}} \varphi_{j k}(x) \mathrm{d} x \\
& \quad+C \sum_{k, j} \int_{-N}^{d} A_{j k} R(x)^{p_{D}^{-}} \varphi_{j k}(x) \mathrm{d} x \\
& \quad+C \sum_{k, j} \int_{-N}^{d} R(x)^{p_{D}^{-}} \varphi_{j k}(x) \mathrm{d} x=: \mathbf{I}_{\mathbf{1}}+\mathbf{I}_{\mathbf{2}}+\mathbf{I}_{\mathbf{3}} .
\end{aligned}
$$

Let $T$ be the following linear operator:

$$
T h(x)=\sum_{k, j} A_{j k} h(x)
$$

and $\varphi(x)=\sum_{k, j} \varphi_{j k}(x)$. Since $\|f v\|_{L^{p(\cdot)}(D)}=1$, if we show that the operator $T$ is bounded from $L^{p_{D}^{-}}((-N, d), \sigma \mathrm{d} x)$ to $L^{p_{D}^{-}}((-N, d), \varphi \mathrm{d} x)$, we obtain

$$
\begin{aligned}
\mathbf{I}_{1} & =C \int_{-N}^{d}\left(T\left(\left(f \sigma^{-1}\right)^{p(\cdot) / p_{D}^{-}}\right)(x)\right)^{p_{D}^{-}} \varphi(x) \mathrm{d} x \\
& \leqslant C \int_{-N}^{d}\left(f(x) \sigma(x)^{-1}\right)^{p(x)} \sigma(x) \mathrm{d} x \\
& \leqslant C \int_{D} f(x)^{p(x)} v(x)^{p(x)} \mathrm{d} x \leqslant C .
\end{aligned}
$$

It is easy to see that $T$ is bounded on $L^{\infty}(D)$. Therefore, we only need to show that $T$ is bounded from $L^{1}((-N, d), \sigma \mathrm{d} x)$ to $L^{1, \infty}((-N, d), \varphi \mathrm{d} x)$ to complete our proof by the Marcinkiewicz interpolation theorem. For arbitrary $\lambda>0$ and each pair $(j, k)$, define

$$
F_{j k}(\lambda):=E_{j k} \cap\{x \in(-N, d): T h(x)>\lambda\} .
$$

Obviously, $F_{j k}(\lambda)$ are pairwise disjoint. Let $c_{j k}(\lambda)$ := $\inf F_{j k}(\lambda)$ and $J_{j k}=J_{j k}(\lambda):=\left[c_{j k}(\lambda), b_{j k}\right)$. It is clear that any two intervals of $\left\{J_{j k}\right\}$ are disjoint or one is contained in the other. By the definition of $J_{j k}$, we also have

$$
\int_{J_{j k}} h(t) \sigma(t) \mathrm{d} t \geqslant \lambda \int_{J_{j k}} \sigma(t) \mathrm{d} t .
$$

Let $\left\{J_{i}\right\}$ be the maximal elements of the family $\left\{J_{j k}\right\}$. These maximal elements exist since the intervals $J_{j k}$ have uniformly bounded length. The intervals $J_{i}$ also satisfy (46). Then, by (9) and (46), we obtain

$$
\begin{aligned}
& \int_{\{x \in(-N, d): T h(x)>\lambda\}} \varphi(x) \mathrm{d} x \\
& =\sum_{i} \sum_{\left\{k, j: J_{i} \supset J_{j k}\right\}} \int_{F_{j k}(\lambda)}\left(\frac{1}{b_{j k}-x} \int_{x}^{b_{j k}} \chi_{J_{i}}(t) \sigma(t) \mathrm{d} t\right)^{p(x)} \\
& \cdot u(x)^{p(x)} \mathrm{d} x \leqslant \sum_{i} \int_{J_{i}} M^{+}\left(\chi_{J_{i}} \sigma\right)(x)^{p(x)} \\
& \cdot u(x)^{p(x)} \mathrm{d} x \leqslant C \sum_{i} \int_{J_{i}} \sigma(x) \mathrm{d} x \leqslant C \sum_{i} \frac{1}{\lambda} \\
& \cdot \int_{J_{i}} h(x) \sigma(x) \mathrm{d} x \leqslant \frac{C}{\lambda} \int_{-N}^{d} h(x) \sigma(x) \mathrm{d} x .
\end{aligned}
$$

This has proved the weak $(1,1)$ inequality for $T$. Hence the estimate for $\mathbf{I}_{1}$ is completed.

Since linear operator $T$ is bounded from $L^{p_{D}^{-}}((-N, d)$, $\sigma \mathrm{d} x)$ to $L^{p_{D}^{-}}((-N, d), \varphi \mathrm{d} x)$, by the second inequality of $(10)$,

$$
\begin{aligned}
\mathbf{I}_{2} & =C \int_{-N}^{d} T R(x)^{p_{D}^{-}} \varphi(x) \mathrm{d} x \\
& \leqslant C \int_{-N}^{d} R(x)^{p_{D}^{-}} \sigma(x) \mathrm{d} x \leqslant C \int_{D} \frac{\sigma(x)}{(e+|x|)^{m p_{D}^{-}}} \mathrm{d} x \\
& \leqslant C .
\end{aligned}
$$


Similarly, by (10), we have

$$
\begin{aligned}
\mathbf{I}_{3} & =C \sum_{k, j} \int_{-N}^{d} \frac{1}{(e+|x|)^{m p_{D}^{-}}}\left(\frac{1}{b_{j k}-x} \int_{x}^{b_{j k}} \sigma(t) \mathrm{d} t\right)^{p(x)} \\
& \cdot u(x)^{p(x)} \chi_{E_{j k}}(x) \mathrm{d} x \leqslant C \int_{-N}^{d}\left(M^{+}\left(\sigma \chi_{(-N, d)}\right)\right. \\
& \cdot(x))^{p(x)} \frac{u(x)^{p(x)}}{(e+|x|)^{m p_{D}^{-}}} \mathrm{d} x \leqslant C \int_{-N}^{d} R(x)^{p_{D}^{-}} \sigma(x) \mathrm{d} x \\
& \leqslant C .
\end{aligned}
$$

Therefore, by (42), (44), (48), and (49), we have

$$
\int_{-N}^{d} M^{+} f(x)^{p(x)} u(x)^{p(x)} \mathrm{d} x \leqslant C,
$$

where $C$ is independent of $N$. Let $N$ tend to infinity and the proof of Theorem 5 is finished.

Theorem 6 can be proved similarly.

Proof of Theorem 7. We can assume $D$ to be a bounded open interval and $f$ to be nonnegative with $\|f v\|_{L^{p(\cdot)}(D)}=1$. It is sufficient to prove that

$$
\int_{D} M^{+} f(x)^{p(x)} u(x)^{p(x)} \mathrm{d} x \leqslant C .
$$

For $k \in \mathbb{Z}$, define

$$
\Omega_{k}=\left\{x \in D: M^{+} f(x)>2^{k}\right\} .
$$

Let $I_{j k}=\left(a_{j k}, b_{j k}\right)$ be the component intervals of $\Omega_{k}$ and $E_{j k}=$ $I_{j k} \backslash \Omega_{k+1}$, where $j$ is an integer. Using the same procedure as (24), we obtain

$$
\begin{aligned}
& \int_{D} M^{+} f(x)^{p(x)} u(x)^{p(x)} \mathrm{d} x \\
& \leqslant 2^{p^{+}} \sum_{k, j} \int_{D} A_{j k}\left(f \sigma^{-1}\right)(x)^{p(x)} \varphi_{j k}(x) \mathrm{d} x,
\end{aligned}
$$

where

$$
\begin{aligned}
& A_{j k} g(x)\left(\frac{1}{\int_{x}^{b_{j k}} \sigma(t) \mathrm{d} t} \int_{x}^{b_{j k}} g(t) \sigma(t) \mathrm{d} t\right) \chi_{E_{j k}}(x), \\
& \varphi_{j k}(x) \\
& \quad=\left(\frac{1}{b_{j k}-x} \int_{x}^{b_{j k}} \sigma(t) \mathrm{d} t\right)^{p(x)} u(x)^{p(x)} \chi_{E_{j k}}(x) .
\end{aligned}
$$

Let $g(x)=f(x) \sigma^{-1}(x), g_{1}(x)=g(x) \chi_{\{x \in D: g(x)>1\}}(x)$ and $g_{2}(x)=g(x) \chi_{\{x \in D: g(x) \leqslant 1\}}(x)$. The estimate for $A_{j k} g_{1}(x)$ is the same as (35). Since $A_{j k} g_{2}(x) \leqslant 1$, we have

$$
\begin{aligned}
A_{j k} g(x)^{p(x)} & \leqslant C\left(A_{j k} g_{1}(x)^{p(x)}+A_{j k} g_{2}(x)^{p(x)}\right) \\
& \leqslant C\left(\left(A_{j k}\left(g^{p(\cdot) / p_{D}^{-}}\right)(x)\right)^{p_{D}^{-}}+1\right) .
\end{aligned}
$$

Combining (53) and (55), we obtain

$$
\begin{aligned}
& \int_{D} M^{+} f(x)^{p(x)} u(x)^{p(x)} \mathrm{d} x \\
& \leqslant C \sum_{k, j} \int_{D}\left(A_{j k}\left(\left(f \sigma^{-1}\right)^{p(\cdot) / p_{D}^{-}}\right)(x)\right)^{p_{D}^{-}} \varphi_{j k}(x) \mathrm{d} x \\
& \quad+C \sum_{k, j} \int_{D} \varphi_{j k}(x) \mathrm{d} x=: \mathbf{I}_{\mathbf{4}}+\mathbf{I}_{\mathbf{5}} .
\end{aligned}
$$

Similarly as $\mathbf{I}_{\mathbf{1}}$ we can estimate $\mathbf{I}_{\mathbf{4}}$ to get $\mathbf{I}_{\mathbf{4}} \leqslant C$. Applying (9) to $\mathbf{I}_{5}$, we have

$$
\begin{aligned}
\mathbf{I}_{5} & =C \sum_{k, j} \int_{D}\left(\frac{1}{b_{j k}-x} \int_{x}^{b_{j k}} \sigma(t) \mathrm{d} t\right)^{p(x)} u(x)^{p(x)} \\
& \cdot \chi_{E_{j k}}(x) \mathrm{d} x \leqslant C \int_{D} M^{+}\left(\sigma \chi_{D}\right)(x)^{p(x)} \\
& \cdot u(x)^{p(x)} \mathrm{d} x \leqslant C \int_{D} \sigma(x) \mathrm{d} x \leqslant C .
\end{aligned}
$$

The Corollary 8 can be obtained by the results of Theorems 5, 6, and 7 directly.

Proof of Corollary 9. Without loss of generality, we can assume that $f$ is nonnegative and bounded with a compact support and $\|f v\|_{p(\cdot)}=1$. Let $H=\mathbb{R} \backslash[-c, c]$. Due to Theorem 7 we only need to prove

$$
\int_{H} M^{+} f(x)^{p(x)} u(x)^{p(x)} \mathrm{d} x \leqslant C .
$$

Let $N>c$ be a positive integer and $D=(-N, c) \cup(c, N)$. For $k \in \mathbb{Z}$, define

$$
\Omega_{k}=\left\{x \in H: M^{+} f(x)>2^{k}\right\} \cap D .
$$

Let $I_{j k}=\left(a_{j k}, b_{j k}\right)$ be the component intervals of $\Omega_{k}$ and $E_{j k}=$ $I_{j k} \backslash \Omega_{k+1}$, where $j$ is an integer. Using the same procedure as (24), we obtain

$$
\begin{aligned}
& \int_{D} M^{+} f(x)^{p(x)} u(x)^{p(x)} \mathrm{d} x \\
& \leqslant 2^{p^{+}} \sum_{k, j} \int_{D} A_{j k}\left(f \sigma^{-1}\right)(x)^{p(x)} \varphi_{j k}(x) \mathrm{d} x,
\end{aligned}
$$

where $A_{j k}$ and $\varphi_{j k}$ have the similar definitions as those in the proof of Theorem 5. Let $g(x)=f(x) \sigma^{-1}(x), g_{1}(x)=$ $g(x) \chi_{\{x \in D: g(x)>1\}}(x)$ and $g_{2}(x)=g(x) \chi_{\{x \in D: g(x) \leqslant 1\}}(x)$. The estimate for $A_{j k} g_{1}(x)$ is also the same as (35). Let $q(x)=$ $p(x) / p^{-}$, then $q(x) \in L H_{\infty}(H)$ and $q(x) \geqslant q_{\infty}=p_{\infty} / p^{-}$for 
$x \in H$. Since $A_{j k} g_{2}(x) \leqslant 1$ for every $j$ and $k$, by the Hölder inequality and Lemma 17 ,

$$
\begin{gathered}
A_{j k} g_{2}(x)^{q(x)} \leqslant A_{j k} g_{2}(x)^{q_{\infty}} \leqslant C\left(\int_{x}^{b_{j k}} \sigma(t) \mathrm{d} t\right)^{-1} \\
\cdot \int_{x}^{b_{j k}} g_{2}(t)^{q_{\infty}} \sigma(t) \mathrm{d} t \leqslant C\left(\int_{x}^{b_{j k}} \sigma(t) \mathrm{d} t\right)^{-1} \\
\cdot\left(\int_{x}^{b_{j k}} g_{2}(t)^{q(t)} \sigma(t) \mathrm{d} t+\int_{x}^{b_{j k}} \frac{\sigma(t)}{\left.(e+|t|)^{m q_{\left(x, b_{j k}\right)}^{-}} \mathrm{d} t\right)}\right. \\
\leqslant C\left(A_{j k}\left(g_{2}^{q(\cdot)}\right)(x)+A_{j k}\left((e+|x|)^{-m}\right)(x)\right) .
\end{gathered}
$$

By (35) and (61), we get

$$
\begin{aligned}
& A_{j k} g(x)^{p(x)} \leqslant C\left(A_{j k} g_{1}(x)^{q(x)}+A_{j k} g_{2}(x)^{q(x)}\right)^{p^{-}} \\
& \quad \leqslant C\left(\left(A_{j k}\left(g^{p(\cdot) / p^{-}}\right)(x)\right)^{p^{-}}\right. \\
& \left.\quad+\left(A_{j k}\left((e+|x|)^{-m}\right)(x)\right)^{p^{-}}\right) .
\end{aligned}
$$

Therefore we have

$$
\begin{aligned}
& \int_{D} M^{+} f(x)^{p(x)} u(x)^{p(x)} \mathrm{d} x \\
& \leqslant C \sum_{k, j} \int_{D}\left(A_{j k}\left(\left(f \sigma^{-1}\right)^{p(\cdot) / p^{-}}\right)(x)\right)^{p^{-}} \varphi_{j k}(x) \mathrm{d} x \\
& \quad+C \sum_{k, j} \int_{D}\left(A_{j k}\left((e+|x|)^{-m}\right)(x)\right)^{p^{-}} \varphi_{j k}(x) \mathrm{d} x \\
& =: \mathbf{I}_{\mathbf{6}}+\mathbf{I}_{\mathbf{7}} .
\end{aligned}
$$

By the similar estimates of $\mathbf{I}_{1}$ and $\mathbf{I}_{2}$, we get $\mathbf{I}_{6} \leqslant C$ and

$$
\begin{aligned}
\mathbf{I}_{7} & \leqslant C \int_{D} \frac{\sigma(x)}{(e+|x|)^{m p^{-}}} \mathrm{d} x \\
& \leqslant C\left(\int_{-N}^{-c} \frac{\sigma(x)}{(e+|x|)^{m p^{-}}} \mathrm{d} x+\int_{c}^{N} \frac{\sigma(x)}{(e+|x|)^{m p^{-}}} \mathrm{d} x\right) \\
& \leqslant C .
\end{aligned}
$$

Let $N$ tend to infinity and the proof is complete.

\section{Applications}

In this section, we assume that $I=(0, b)$ with $0<b<+\infty$. Let

$$
\begin{array}{cc}
\mathscr{R}_{\alpha(\cdot)} f(x)=\int_{0}^{x} f(t)(x-t)^{\alpha(x)-1} \mathrm{~d} t, & x \in(0, b), \\
\mathscr{W}_{\alpha(\cdot)} f(x)=\int_{x}^{b} f(t)(x-t)^{\alpha(x)-1} \mathrm{~d} t, & x \in(0, b),
\end{array}
$$

where $0<\alpha(x)<1$. If $\alpha(x)$ is a constant function, $\mathscr{R}_{\alpha}$ is the classical Riemann-Liouville operator and $\mathscr{W}_{\alpha}$ is the classical Weyl operator. In [17], Andersen and Sawyer obtained the two weighted norm inequalities of $\mathscr{R}_{\alpha}$ and $\mathscr{W}_{\alpha}$ from $L^{p}(\mathbb{R}, u \mathrm{~d} x)$ to $L^{q}(\mathbb{R}, v \mathrm{~d} x)$. Other results about $\mathscr{R}_{\alpha}$ and $\mathscr{W}_{\alpha}$ on $L^{p}$ can be seen in [18-21] and so forth.

Edmunds et al. [9] studied the boundedness of $\mathscr{R}_{\alpha}, \mathscr{W}_{\alpha}$, $\mathscr{R}_{\alpha(\cdot)}$, and $\mathscr{W}_{\alpha(\cdot)}$ in variable Lebesgue spaces. Kokilashvili et al. [11] discussed the two weighted norm inequalities of $\mathscr{R}_{\alpha}$ and $\mathscr{W}_{\alpha}$ from $L_{w}^{p(\cdot)}\left(\mathbb{R}_{+}\right)$to $L_{v}^{q(\cdot)}\left(\mathbb{R}_{+}\right)$. In this section, we will discuss the two-weight inequalities of $\mathscr{R}_{\alpha(\cdot)}$ and $\mathscr{W}_{\alpha(\cdot)}$ in $L^{p(\cdot)}(I)$.

Definition 18 (see [9]). Given $p(\cdot): E \rightarrow[1,+\infty$ ), we say that $p(\cdot) \in \mathscr{P}^{-}(E)$ if there exists a constant $C>0$ such that for all $x, y \in E$, with $0<x-y \leqslant 1 / 2$,

$$
p(x)-p(y) \leqslant \frac{C}{-\ln (x-y)} .
$$

We also say that $p(\cdot) \in \mathscr{P}^{+}(E)$ if there exists a constant $C>0$ such that for all $x, y \in E$ with $0<y-x \leqslant 1 / 2$,

$$
p(x)-p(y) \leqslant \frac{C}{-\ln (y-x)} .
$$

Our results in this section are the following theorems:

Theorem 19. It is given that $p(\cdot): I \rightarrow[1,+\infty)$ such that $1<$ $p_{I}^{-} \leqslant p_{I}^{+}<+\infty$ and $0<\alpha_{I}^{-} \leqslant \alpha(x)<1 / p_{I}^{+}$. If $\left(u^{q(\cdot) / p(\cdot)}, v\right) \epsilon$ $S_{p(\cdot)}^{+}(I)$ and $p(\cdot) \in\left(\mathscr{P}_{\sigma}^{+}(I) \cap \mathscr{P}^{+}(I)\right)$, where $\sigma(x)=v(x)^{-p^{\prime}(x)}$ and $q(x)=p(x) /(1-\alpha(x) p(x))$, then

$$
\left\|\left(\mathscr{W}_{\alpha(\cdot)} f\right) u\right\|_{L^{q(\cdot)}(I)} \leqslant C\|f v\|_{L^{p(\cdot)}(I)} .
$$

Theorem 20. Given $p(\cdot): I \rightarrow[1,+\infty)$ such that $1<p_{I}^{-} \leqslant$ $p_{I}^{+}<+\infty$ and $0<\alpha_{I}^{-} \leqslant \alpha(x)<1 / p_{I}^{+}$. If $\left(u^{q(\cdot) / p(\cdot)}, v\right) \in S_{p(\cdot)}^{-}(I)$ and $p(\cdot) \in\left(\mathscr{P}_{\sigma}^{-}(I) \cap \mathscr{P}^{-}(I)\right)$, where $\sigma(x)=v(x)^{-p^{\prime}(x)}$ and $q(x)=p(x) /(1-\alpha(x) p(x))$, then

$$
\left\|\left(\mathscr{R}_{\alpha(\cdot)} f\right) u\right\|_{L^{q(\cdot)}(I)} \leqslant C\|f v\|_{L^{p(\cdot)}(I)} .
$$

Remark 21. The sets $\mathscr{P}_{\sigma}^{+}(I) \cap \mathscr{P}^{+}(I)$ and $\mathscr{P}_{\sigma}^{-}(I) \cap \mathscr{P}^{-}(I)$ are not empty. Let $p(\cdot) \in \mathscr{P}^{+}(I)$ (resp., $p(\cdot) \in \mathscr{P}^{-}(I)$ ). If $\sigma$ is lower Ahlfors $Q$-regular $(Q>0)$, which means there exists a constant $C=C(Q)>0$ such that $\int_{a}^{b} \sigma(t) \mathrm{d} t \geqslant C(b-a)^{\mathrm{Q}}$ for every bounded interval $(a, b) \subset I$, then $p(\cdot) \in \mathscr{P}_{\sigma}^{+}(I)$ (resp., $\left.p(\cdot) \in \mathscr{P}_{\sigma}^{-}(I)\right)$.

In order to prove Theorem 19, we need the following lemma.

Lemma 22 (see [9]). It is given that $p(\cdot) \in \mathscr{P}^{+}(I), 0<\alpha_{I}^{-} \leqslant$ $\alpha(x)<1 / p_{I}^{+}$, and $q(x)=p(x) /(1-\alpha(x) p(x))$. If $\|f\|_{L^{p(\cdot)}(I)} \leqslant 1$, then there exists a positive constant $C$ depending only on $p(\cdot)$ and $\alpha(\cdot)$ such that

$$
\mathscr{W}_{\alpha(\cdot)}(|f|)(x) \leqslant C\left[M^{+} f(x)\right]^{p(x) / q(x)}, \quad x \in I .
$$


Proof of Theorem 19. By the homogeneity of norm, we can assume that $\|f v\|_{L^{p(\cdot)}(I)} \leqslant 1$ and $\|f\|_{L^{p(\cdot)}(I)} \leqslant 1$. It is sufficient to prove

$$
\int_{I}\left(\mathscr{W}_{\alpha(x)} f\right)(x)^{q(x)} u(x)^{q(x)} \mathrm{d} x \leqslant C .
$$

Applying (70) and Theorem 7 we have

$$
\begin{aligned}
& \int_{I}\left(\mathscr{W}_{\alpha(x)} f\right)(x)^{q(x)} u(x)^{q(x)} \mathrm{d} x \\
& \quad \leqslant C \int_{I}\left(M^{+} f\right)(x)^{p(x)}\left(u(x)^{q(x) / p(x)}\right)^{p(x)} \mathrm{d} x \leqslant C .
\end{aligned}
$$

Using the similar proving method as that of Lemma 3.1 in [9], we can prove the following lemma.

Lemma 23. It is given that $p(\cdot) \in \mathscr{P}^{-}(I), 0<\alpha_{I}^{-} \leqslant \alpha(x)<$ $1 / p_{I}^{+}$, and $q(x)=p(x) /(1-\alpha(x) p(x))$. If $\|f\|_{L^{p(\cdot)}(I)} \leqslant 1$, then there exists a positive constant $C$ depending only on $p(\cdot)$ and $\alpha(\cdot)$ such that

$$
\mathscr{R}_{\alpha(\cdot)}(|f|)(x) \leqslant C\left[M^{-} f(x)\right]^{p(x) / q(x)}, \quad x \in I .
$$

By this lemma and the two weighted results of $M^{-}$, we can get the result of Theorem 20 directly.

\section{Competing Interests}

The authors declare that there are no competing interests regarding the publication of this paper.

\section{Acknowledgments}

This research is supported by NNSF-China (Grants nos. 11171345 and 51234005).

\section{References}

[1] E. Sawyer, "Weighted inequalities for the one-sided HardyLittlewood maximal functions," Transactions of the American Mathematical Society, vol. 297, no. 1, pp. 53-61, 1986.

[2] F. J. Martín-Reyes, P. Ortega Salvador, and A. de la Torre, "Weighted inequalities for one-sided maximal functions," Transactions of the American Mathematical Society, vol. 319, no. 2, pp. 517-534, 1990.

[3] F. J. Martín-Reyes, "New proofs of weighted inequalities for the one-sided Hardy-Littlewood maximal functions," Proceedings of the American Mathematical Society, vol. 117, no. 3, pp. 691-698, 1993.

[4] D. V. Cruz-Uribe and A. Fiorenza, Variable Lebesgue Spaces: Foundations and Harmonic Analysis, Applied and Numerical Harmonic Analysis, Springer, Basel, Switzerland, 2013.

[5] X. Fan and D. Zhao, "On the spaces $L^{p(x)}(\Omega)$ and $W^{m, p(x)}(\Omega)$," Journal of Mathematical Analysis and Applications, vol. 263, no. 2, pp. 424-446, 2001.

[6] O. Kovácik and J. Rákosník, "On spaces $L^{p(x)}$ and $W^{k, p(x)}$," Czechoslovak Mathematical Journal, vol. 41, no. 4, pp. 592-618, 1991.
[7] L. Diening, P. Harjulehto, P. Hästö, and M. Růžička, Lebesgue and Sobolev Spaces with Variable Exponents, vol. 2017 of Lecture Notes in Mathematics, Springer, Berlin, Germany, 2011.

[8] J. Musielak, Orlicz Spaces and Modular Spaces, vol. 1034, no 4 of Lecture Notes in Mathematics, Springer, Berlin, Germany, 1983.

[9] D. E. Edmunds, V. Kokilashvili, and A. Meskhi, "One-sided operators in $L^{p(x)}$ spaces," Mathematische Nachrichten, vol. 281, no. 11, pp. 1525-1548, 2008.

[10] M. I. Aguilar Cañestro and P. Ortega Salvador, "Weighted weak type inequalities with variable exponents for Hardy and maximal operators," Proceedings of the Japan Academy, Series A, Mathematical Sciences, vol. 82, no. 8, pp. 126-130, 2006.

[11] V. Kokilashvili, A. Meskhi, and M. Sarwar, "One and two weight estimates for one-sided operators in $L^{p(x)}$ spaces," Eurasian Mathematical Journal, vol. 1, no. 1, pp. 73-110, 2010.

[12] E. T. Sawyer, "A characterization of a two-weight norm inequality for maximal operators," Studia Mathematica, vol. 75, no. 1, pp. 1-11, 1982.

[13] V. Kokilashvili and A. Meskhi, "Two-weight inequalities for fractional maximal functions and singular integrals in $L^{p(x)}$ spaces," Journal of Mathematical Sciences, vol. 173, no. 6, pp. 656-673, 2011.

[14] F. I. Mamedov and Y. Zeren, "On a two-weighted estimation of maximal operator in the Lebesgue space with variable exponent," Annali di Matematica Pura ed Applicata, vol. 190, no. 2, pp. 263-275, 2011.

[15] F. Mamedov and Y. Zeren, "On boundedness of fractional maximal operator in weighted $L^{p(x)}$ spaces," Mathematical Ineqalities Applications, vol. 19, no. 1, pp. 1-14, 2016.

[16] D. Cruz-Uribe, A. Fiorenza, and C. J. Neugebauer, "Weighted norm inequalities for the maximal operator on variable Lebesgue spaces," Journal of Mathematical Analysis and Applications, vol. 394, no. 2, pp. 744-760, 2012.

[17] K. F. Andersen and E. T. Sawyer, "Weighted norm inequalities for the Riemann-Liouville and Weyl fractional integral operators," Transactions of the American Mathematical Society, vol. 308, no. 2, pp. 547-558, 1988.

[18] F. J. Martín-Reyes and E. Sawyer, "Weighted inequalities for Riemann-Liouville fractional integrals of order one and greater," Proceedings of the American Mathematical Society, vol. 106, no. 3, pp. 727-733, 1989.

[19] A. Meskhi, "Solution of some weight problems for the RiemannLiouville and Weyl operators," Georgian Mathematical Journal, vol. 5, no. 6, pp. 565-574, 1998.

[20] M. Lorente and M. S. Riveros, "Weights for commutators of the one-sided discrete suqare function, the Weyl fractional integral and other one-sided operators," Proceedings of the Royal Society of Edinburgh A, vol. 135, pp. 845-862, 2005.

[21] Z. W. Fu and Y. Lin, "Weighted boundedness for cummutators of one-sided operators," Acta Mathematica Sinica, Chinese Series, vol. 54, no. 5, pp. 705-714, 2011. 


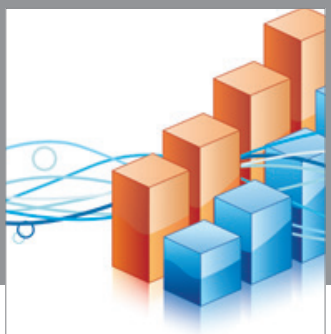

Advances in

Operations Research

vatem alat4

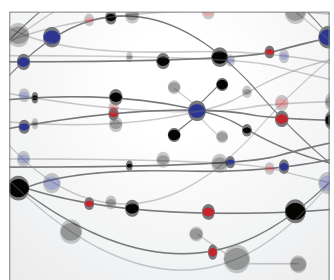

\section{The Scientific} World Journal
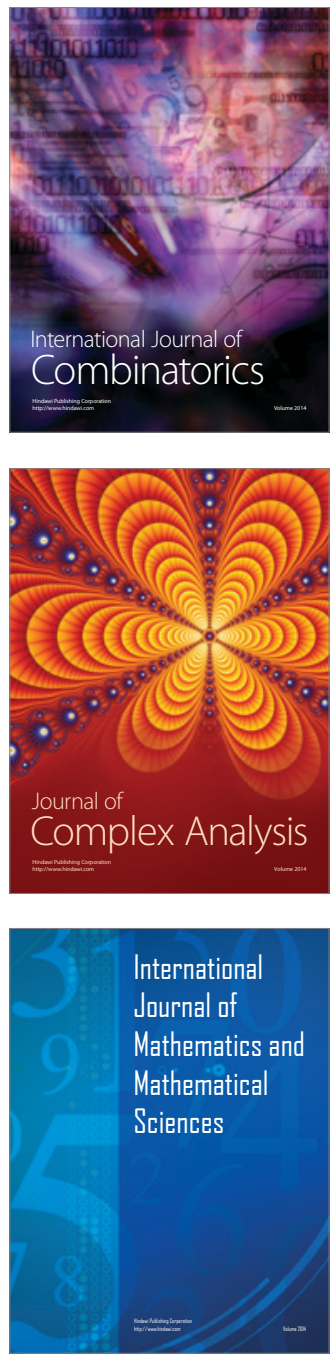
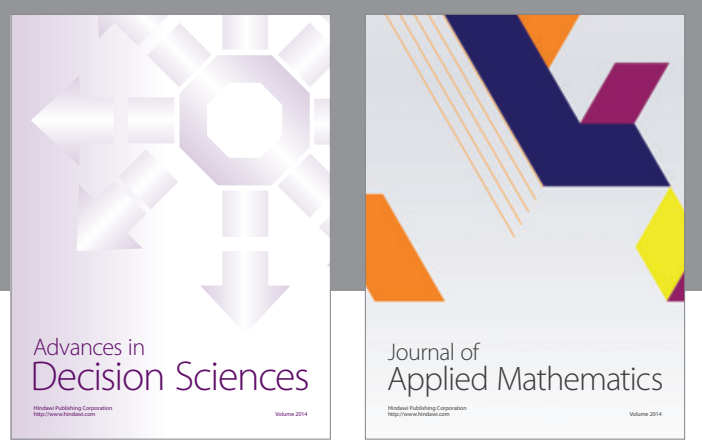

Algebra

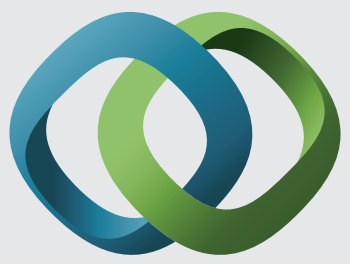

\section{Hindawi}

Submit your manuscripts at

http://www.hindawi.com
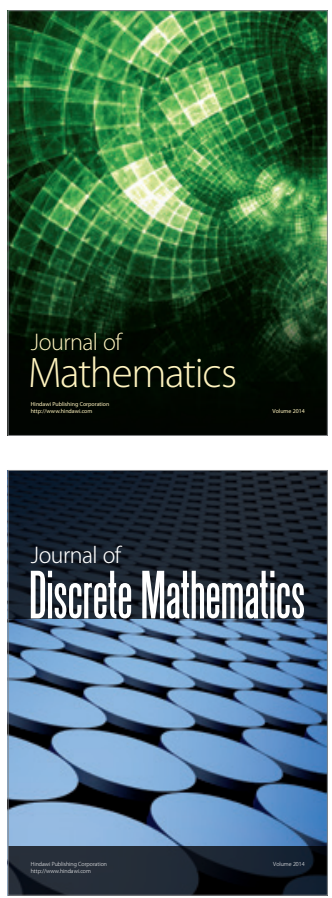

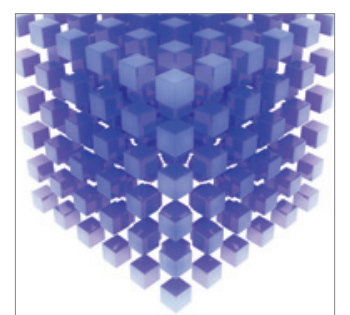

Mathematical Problems in Engineering
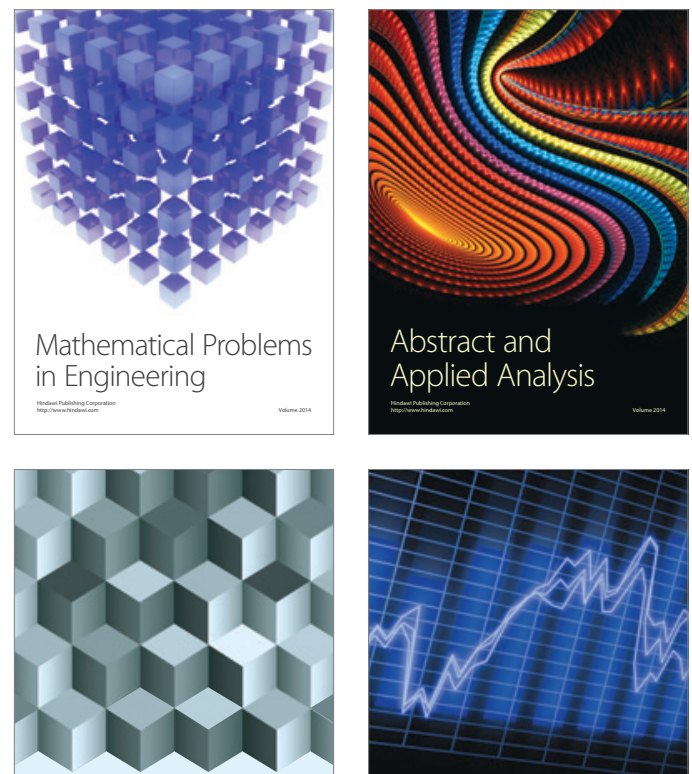

Journal of

Function Spaces

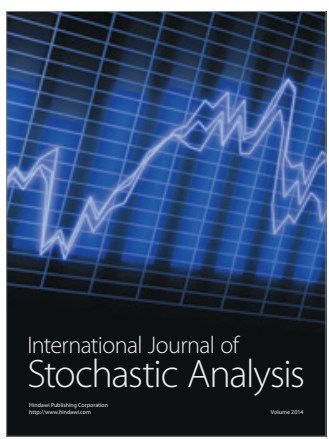

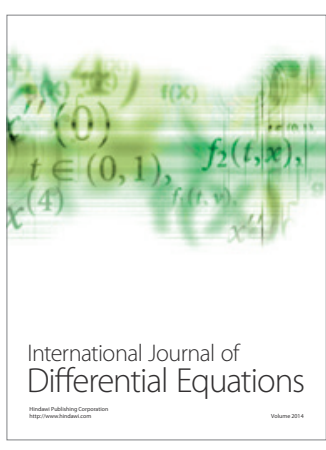
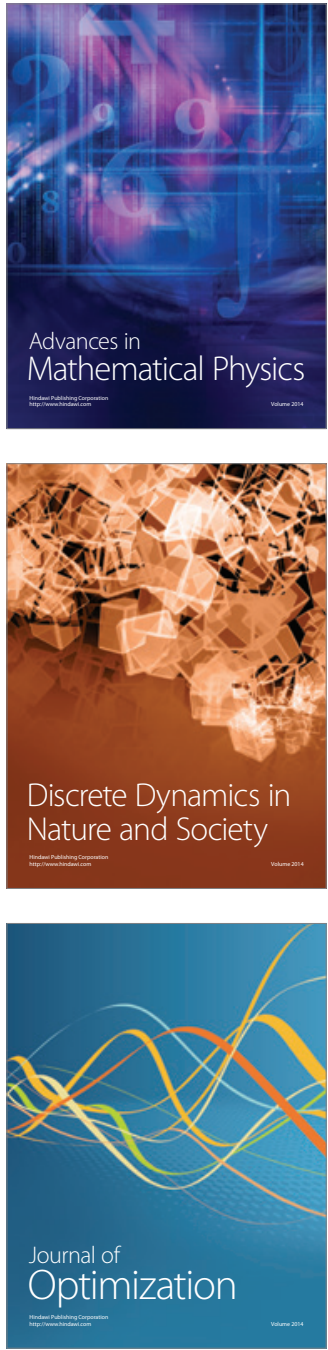